\title{
Reagent Preparation and Storage for Amplification of Microarray Hybridization Targets with a Fully Automated System
}

\author{
Mingjie Zhou, ${ }^{1}$ Jon Marlowe, ${ }^{2}$ Jaime Graves, ${ }^{1}$ Jason Dahl, ${ }^{1}$ Zackery Riley, ${ }^{3}$ Lena Tian, ${ }^{4}$ \\ Sven Duenwald, ${ }^{1}$ George Tokiwa, ${ }^{1}$ and Thomas L. Fare ${ }^{1}$
}

\begin{abstract}
The advent of automated systems for gene expression profiling has accentuated the need for the development of convenient and cost-effective methods for reagent preparation. We have developed a method for the preparation and storage of pre-aliquoted cocktail plates that contain all reagents required for amplification of nucleic acid by reverse transcription and in vitro transcription reactions. Plates can be stored at $-80^{\circ} \mathrm{C}$ for at least 1 month and kept in a hotel at $4^{\circ} \mathrm{C}$ for at least $24 \mathrm{~h}$ prior to use. Microarray data quality generated from these pre-aliquoted reagent plates is not statistically different between cRNA amplified with stored cocktails and cRNA amplified with freshly prepared cocktails. Deployment of pre-aliquoted, stored cocktail plates in a fully automated system not only increases the throughput of amplifying cRNA targets from thousands of RNA samples, but could also considerably reduce reagent costs and potentially improve process robustness.
\end{abstract}

\section{Introduction}

$\mathbf{T}$ HE DEVELOPMENT of microarray technology makes it possible to measure the expression of thousands of genes in the same hybridization experiment on a single array. ${ }^{1}$ Microarray profiling technology has become increasingly popular for biological research and medical applications, particularly in the process of drug discovery, development, and validation. In all microarray platforms, sample preparation is a critical step in using this technology. Even with high-quality total RNA or mRNA, it is still a challenge to obtain enough target for microarray hybridization to profile each gene in the original sample. The in vitro transcription (IVT) of cDNA derived from the reverse transcription (RT) of total RNA or mRNA (commonly called the RT-IVT method) is the method for global mRNA amplification ${ }^{2}$ to be used in this work.
Throughout the various phases of drug discovery and development, thousands of RNA samples could be collected for microarray-based experiments. In order to utilize microarrays to investigate differential expression profiling for such experiments, a high throughput method for the preparation of cRNA or cDNA is required. Compared to conventional HTS assays, the preparation of cRNA or cDNA targets from total RNA or mRNA is more complicated in terms of procedures, instruments, and particularly the duration of the process. While a manual procedure for the RT-IVT method can provide the high-quality cRNA target for microarray hybridization with a small set of mRNA or total RNA samples, it is difficult to generate a consistent quality of cRNA target from hundreds or thousands of individual RNA samples reproducibly. Moreover, most of the variability in cRNA preparation seems to be due to minor deviations inherent

\footnotetext{
${ }^{1}$ Rosetta Inpharmatics LLC; ${ }^{3}$ Methods Development, The Allen Institute; and ${ }^{4}$ Department of Molecular \& Cellular Discovery, Zymogenetics, Seattle, WA.

${ }^{2}$ Systems Development, Roche Molecular Diagnostics, Pleasanton, CA.

Rosetta Inpharmatics LLC is a wholly owned subsidiary of Merck \& Co. Inc.
}

ABBREVIATIONS: CV, coefficient of variation; FAS, fully automated system; IVT, in vitro transcription; RT, reverse transcription; ROC, receiver operating characteristic; SAP, semiautomated procedure. 
in reagents or in manual procedures performed by different persons.

A semiautomated procedure (SAP) was developed by Rosetta Inpharmatics LLC (Seattle, WA) to prepare cDNA and cRNA target for microarray hybridization. ${ }^{3}$ While this method addressed the throughput and quality challenge of cDNA and cRNA target preparation from a large number of samples, it is clear that further improvements in quality and efficiency can be achieved by industrializing reagent preparation and fully automating all procedures.

Using commercially available robotic instruments and proprietary software developed by Rosetta, a fully automated system (FAS) was designed to prepare cDNA and cRNA targets for microarray hybridization. The design goal for the FAS is to process multiple plates of nucleic acid samples in a walk-away manner. Because of the nature of the RT-IVT procedure, the last plate could be initiated a few hours after the first plate in a typical FAS experimental run with multiple plates of samples. Thus, the FAS method requires all reagents be prepared prior to the initiation of RT-IVT protocol and stored in automation-friendly plates. As described in Fig. 1, the prealiquoted cocktail plates are stored in and fed from reagent carousel hotels to Biomek ${ }^{\circledR}$ FX (Beckman Coulter, Fullerton, $\mathrm{CA}$ ) robot decks to serve as the surrogate for an FAS.

Like the manual procedure, the SAP method utilizes reagent plates that were prepared fresh and used within a short period of time. Hence, stability of pre-aliquoted reagent plates would become the next advance towards an FAS for nucleic acid amplification. To this end, a method for the storage of ready-to-use reagent plates was developed.

We tested the stability of cocktail plates under different conditions and found that stored cocktail plates retained their enzymatic activities at $4^{\circ} \mathrm{C}$ for up to $24 \mathrm{~h}$ following storage at $-80^{\circ} \mathrm{C}$ for at least 1 month. (Throughout this paper, we refer to the cocktail plate as the reagent plate that contains all reagents required for RT or IVT reaction, the stored cocktail plate as the reagent plate that was pre-aliquoted and stored under specified conditions, and the fresh cocktail plate as the reagent plate that was prepared immediately before the intended reaction and used within $1 \mathrm{~h}$.) Data generated using stored cocktail plates had the same quality as that obtained from the freshly prepared cocktails as determined by cDNA and cRNA yields from RT and IVT reactions, and as assessed by microarray experiments in detecting the differential expression between different total RNA samples. The reagent cocktail plates should not only enable the preparation of cRNA or cDNA target with the FAS, but would also significantly reduce the reagent cost.

\section{Materials and Methods}

\section{Microarrays and reagents}

Microarrays were acquired from Agilent (San Jose, CA). Total RNA derived from K562 and Jurkat cell lines was purchased from Ambion (Austin, TX). Cy3 and Cy5 dyes and RNAguard ${ }^{\mathrm{TM}}$ were obtained from Amersham

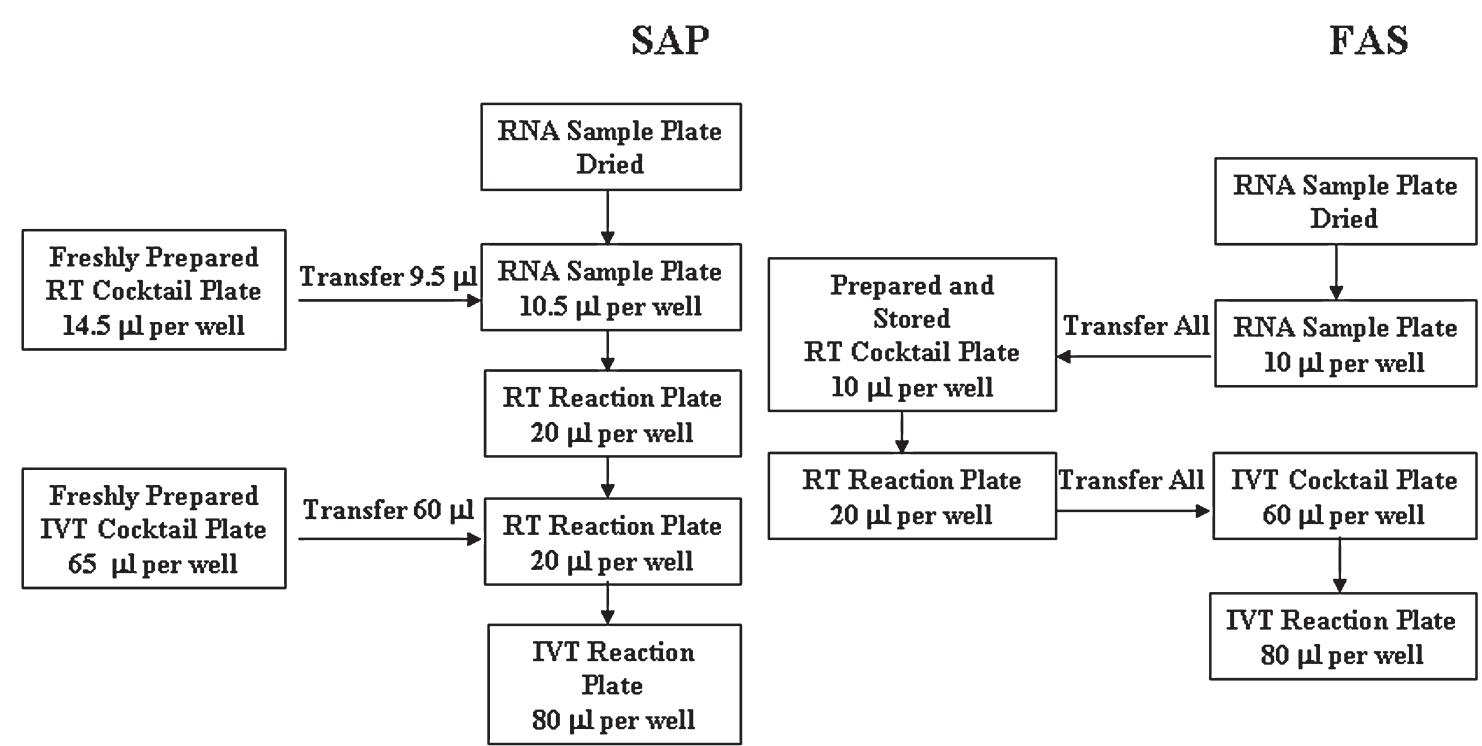

FIG. 1. Flow chart of reagents transfer in RT-IVT reactions. The SAP and FAS methods differed in the preparation of reagent cocktail plates and the reagent transferring procedure. 
(Little Chalfont, Buckinghamshire, UK). Reverse transcriptase derived from Moloney murine leukemia virus and T7 RNA polymerase were from Epicenter (Madison, WI). Superscript ${ }^{\mathrm{TM}}$ II was from Invitrogen Life Technologies (Carlsbad, CA). Pyrophosphatase was obtained from New England Biolabs (Beverly, MA). RNeasy ${ }^{\circledR}$ 96well plates were purchased from Qiagen (Venlo, The Netherlands). The Biomek FX automation workstation was from Beckman Coulter.

\section{Preparation and storage of cocktail plates}

The RT reaction cocktail was first assembled with Moloney murine leukemia virus reverse transcriptase at $2.5 \mathrm{U} / \mu \mathrm{l}$, RNAguard at $5 \mathrm{U} / \mu \mathrm{l}$, random hexamer at 1.0 $\mathrm{ng} / \mu \mathrm{l}$ in buffer containing $50 \mathrm{~m} M$ Tris- $\mathrm{HCl}$ (pH 8.3), 75 $\mathrm{m} M \mathrm{KCl}, 3 \mathrm{~m} M \mathrm{MgCl}_{2}, 10 \mathrm{~m} M$ dithiothreitol, and 0.5 $\mathrm{m} M$ deoxynucleotide triphosphates. After aliquots of 10 $\mu \mathrm{l}$ of RT cocktail or $60 \mu \mathrm{l}$ of IVT cocktail were dispensed to each well of a 96-well microtiter plate (Island Scientific, Bainbridge Island, WA), the plate was sealed by an automated plate sealer (ABgene, Epsom, UK). The sealed plates were stored at $4^{\circ} \mathrm{C}$ for periods of time from 4 to $24 \mathrm{~h}$, at $-20^{\circ} \mathrm{C}$ for $24 \mathrm{~h}$ to 1 week, or at $-80^{\circ} \mathrm{C}$ from 1 day to 1 month. The IVT reaction cocktail was prepared with T7 polymerase at $25 \mathrm{U} / \mu \mathrm{l}$, RNAguard at $0.25 \mathrm{U} / \mu \mathrm{l}$, and pyrophosphatase at $0.015 \mathrm{U} / \mu \mathrm{l}$ in buffer containing $40 \mathrm{~m} M$ Tris- $\mathrm{HCl}$ (pH 7.5), $10 \mathrm{~m} M \mathrm{NaCl}, 14.25 \mathrm{~m} M$ $\mathrm{MgCl}_{2}, 7.5 \mathrm{~m} M$ dithiothreitol, $2.5 \mathrm{~m} M$ each ATP, CTP, and GTP, $1.88 \mathrm{~m} M$ UTP, and $0.6 \mathrm{~m} M$ aminoacyl-UTP. The preparation and storage of IVT reaction cocktail were the same as those for the RT reaction cocktail.

\section{Sample preparation, hybridization, and data analysis}

Amplification of the total RNA to create cRNA was performed according to the SAP as detailed by Hughes et al. ${ }^{3}$ For the FAS method, the RT-IVT procedure was modified from the SAP method (Fig. 1). Briefly, all reagents and sample plates were loaded into the corresponding reagent hotel $\left(\right.$ Cytomat $\left.^{\circledR}\right)$, Kendro Heraeus, Asheville, NC) at $4^{\circ} \mathrm{C}$ before the start of the RT-IVT process. The procedure started with the rehydration of the RNA sample plate with $10 \mu \mathrm{l}$ of water, followed by denaturing the sample at $60^{\circ} \mathrm{C}$ in a thermocycler $\left(\right.$ Tetrad $^{\circledR}$ thermal cycler PTC-100, MJ Research, Ramsey, MN) for 10 min and transfer of the denatured RNA sample into the RT cocktail plate. The RT reaction proceeded at $40^{\circ} \mathrm{C}$ for $2 \mathrm{~h}$ followed by inactivation of the RT enzyme at $70^{\circ} \mathrm{C}$ for $10 \mathrm{~min}$ and then transfer of the RT reaction mixture into the IVT cocktail plate. The IVT reaction occurred at $40^{\circ} \mathrm{C}$ for $16 \mathrm{~h}$. The resulting cRNA target was purified from the IVT reaction mixture with an RNeasy plate-based method. The cRNA and cDNA concentrations were measured by the standard ultraviolet absorption method with a microplate reader (SpectraMax 190,
Molecular Device, Sunnyvale, CA). The purified cRNAs were labeled with $\mathrm{Cy} 3$ and $\mathrm{Cy} 5$ to form fluor-reversed pairs as described. ${ }^{3}$ Microarray hybridizations, scanning of the slides, image analysis, and data analysis were processed as described by Marton et al. ${ }^{4}$

\section{Results and Discussion}

To operate the FAS efficiently, it was essential that all reagents be stored in automation-friendly plates and loaded into a reagent carousel hotel up to $12 \mathrm{~h}$ prior to the initiation of experimental protocols. As a design goal to process multiple plates of nucleic acid sample preparation, we set the requirement that all reagents must be stored in a 96- or 384-well plate and must be stable at $4^{\circ} \mathrm{C}$ in a reagent hotel for up to $24 \mathrm{~h}$. For the bulk preparation of reagent plates and operational flexibility of nucleic acid amplification, it was desirable that the pre-aliquoted cocktail plates be stable under a suitable condition over a time period of 1 week to 1 month.

\section{Conditions for storage of cocktail plates}

The development of a method for storing cocktail plates began with facilities commonly available in a molecular biology research laboratory. The RT and IVT reaction cocktails were prepared in bulk and aliquoted into a 96-well plate. After sealing, the cocktail plates were stored at $4^{\circ} \mathrm{C},-20^{\circ} \mathrm{C}$, or $-80^{\circ} \mathrm{C}$. The performance of stored RT and IVT cocktails was evaluated in RT-IVT experiments in comparison with the fresh cocktails. One critical concern for the storage of RT and IVT reaction cocktails with enzymes was the possibility of losing enzymatic activity during the storage, which could lead to lower cRNA yield from the amplification experiment. As shown in Table 1, cRNA yields were similar among experiments with different combinations of RT and IVT reaction cocktails under indicated storage conditions. As reported in the literature, the coefficient of variation (CV) of cRNA yields was usually within $20 \%$ from a typical amplification experiment of multiple samples when it was performed with the same set of reagents and procedures. $^{4,5}$ When each experiment with reagent cocktails stored at different conditions was analyzed separately, the CV of cRNA yield ranged from $2.2 \%$ to $10.4 \%$ (Table 1). When 13 experiments were analyzed together, the calculated CV was $7.0 \%$. Since the CVs of cRNA yield from the average of each experiment and the average of all experiments were within the expected range of variation for an amplification experiment performed with the reagent cocktails prepared at the same condition, ${ }^{4,5}$ we concluded that storage of reagent cocktails did not negatively affect the cRNA yield. Considering the operational simplicity 
Table 1. Comparison of cRNA Yields in Experiments with Complete Cocktails Stored Under Different Conditions

\begin{tabular}{|c|c|c|c|c|}
\hline Experiment & $R T$ cocktail & IVT cocktail & $c R N A$ yields $(\mu g)$ & $C V(\%)$ \\
\hline 1 & Fresh & Fresh & $40.17 \pm 4.17$ & 10.4 \\
\hline 2 & Fresh & $4^{\circ} \mathrm{C}$ for $24 \mathrm{~h}$ & $42.52 \pm 2.84$ & 6.7 \\
\hline 3 & $4^{\circ} \mathrm{C}$ for $24 \mathrm{~h}$ & Fresh & $43.10 \pm 1.36$ & 3.2 \\
\hline 4 & $4^{\circ} \mathrm{C}$ for $24 \mathrm{~h}$ & $4^{\circ} \mathrm{C}$ for $24 \mathrm{~h}$ & $44.22 \pm 1.58$ & 3.6 \\
\hline 5 & $-80^{\circ} \mathrm{C}$ for $24 \mathrm{~h}$ & Fresh & $44.2 \pm 1.84$ & 4.2 \\
\hline 6 & Fresh & $-80^{\circ} \mathrm{C}$ for $24 \mathrm{~h}$ & $44.50 \pm 3.11$ & 7.0 \\
\hline 7 & $-80^{\circ} \mathrm{C}$ for $24 \mathrm{~h}$ & $-80^{\circ} \mathrm{C}$ for $24 \mathrm{~h}$ & $44.14 \pm 2.01$ & 4.6 \\
\hline 8 & $-80^{\circ} \mathrm{C}$ for 7 days & Fresh & $39.94 \pm 0.89$ & 2.2 \\
\hline 9 & Fresh & $-80^{\circ} \mathrm{C}$ for 7 days & $45.42 \pm 2.19$ & 4.8 \\
\hline 10 & $-80^{\circ} \mathrm{C}$ for 7 days & $-80^{\circ} \mathrm{C}$ for 7 days & $39.73 \pm 1.54$ & 3.9 \\
\hline 11 & $\begin{array}{l}-80^{\circ} \mathrm{C} \text { for } 7 \text { days } \\
\text { and } 4^{\circ} \mathrm{C} \text { for } 24 \mathrm{~h}\end{array}$ & Fresh & $39.89 \pm 2.39$ & 6.0 \\
\hline 12 & Fresh & $\begin{array}{l}-80^{\circ} \mathrm{C} \text { for } 7 \text { days } \\
\text { and } 4^{\circ} \mathrm{C} \text { for } 24 \mathrm{~h}\end{array}$ & $40.83 \pm 1.45$ & 3.5 \\
\hline 13 & $\begin{array}{l}-80^{\circ} \mathrm{C} \text { for } 7 \text { days } \\
\text { and } 4^{\circ} \mathrm{C} \text { for } 24 \mathrm{~h}\end{array}$ & $\begin{array}{l}-80^{\circ} \mathrm{C} \text { for } 7 \text { days } \\
\text { and } 4^{\circ} \mathrm{C} \text { for } 24 \mathrm{~h}\end{array}$ & $39.51 \pm 1.32$ & 3.3 \\
\hline
\end{tabular}

The performance of RT and IVT complete cocktails was evaluated in reactions using the SAP (see Materials and Methods). The data were presented as mean and SD $(n=8)$ for each experiment. The mean and SD of all experiments were 42.17 and 2.98 $\mu \mathrm{g}$, respectively.

and the demonstrated feasibility of a relatively long storage time, we chose $-80^{\circ} \mathrm{C}$ as the storage condition for cocktail plates.

\section{Stability of RT and IVT cocktails at $-80^{\circ} \mathrm{C}$}

Considering factors of sample throughput and the fluctuation of number of samples on a daily basis, we tested the stability of the stored cocktails at $-80^{\circ} \mathrm{C}$ over intervals of $24 \mathrm{~h}$ to 1 month. As shown in Fig. 2A, the difference of cRNA yields among different RT-IVT exper- iments was within the variation of a typical amplification experiment of multiple samples. ${ }^{4,5}$ Specifically, the CVs of cRNA yields ranged from $6.7 \%$ to $13.1 \%$ for Jurkat cells and from $4.8 \%$ to $8.8 \%$ for K562 cells. While the CV at $13.1 \%$ for cRNA yields of Jurkat cells from the experiment with cocktails stored at $-80^{\circ} \mathrm{C}$ for a month was slightly higher than the CV at $10.1 \%$ from the experiment with fresh cocktails, the CVs of cRNA yields of K562 cells were nearly the same for the experiment with fresh cocktails $(\mathrm{CV}=8.8 \%)$ and the experiment with cocktails stored at $-80^{\circ} \mathrm{C}$ for a month $(\mathrm{CV}=8.4 \%)$.
A

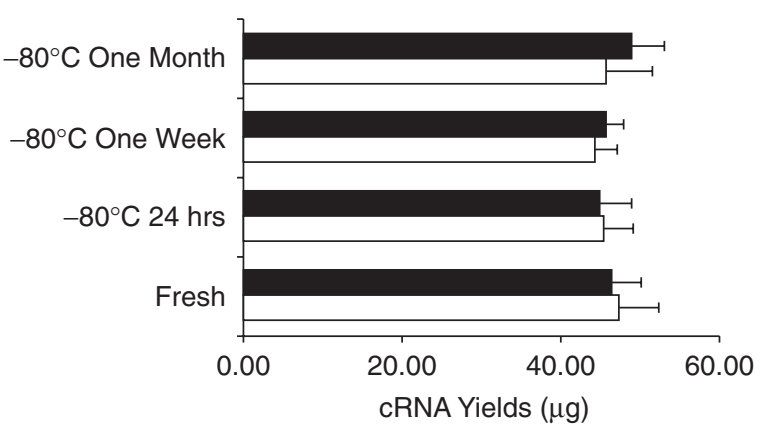

B

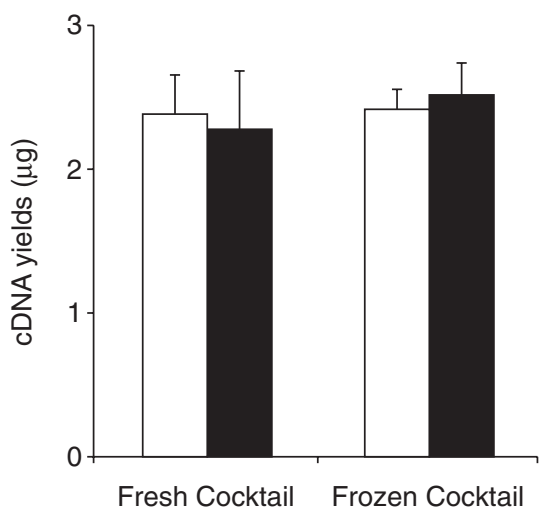

FIG. 2. Stability of stored cocktails. Three sets of RT and IVT cocktail plates were separately prepared, and each was stored at $-80^{\circ} \mathrm{C}$ for 1 month, 1 week, and $24 \mathrm{~h}$ before the RT-IVT experiments were conducted. (A) Total RNAs of K562 ( $\left.\mathbf{\square}\right)$ and Jurkat $(\square)$ cells were processed in RT-IVT reactions with the FAS. The cRNA yields are presented as mean and SD $(n=16)$ for each experiment. (B) Three micrograms of cRNA was used as the input material to generate cDNA in the RT reaction with the SAP. The RT cocktail was stored at $-20^{\circ} \mathrm{C}$ for 2 weeks. Each experiment has 32 replicates. 
The stored RT cocktail was also tested to generate the first-strand cDNA target for the microarray hybridization. The resulting cDNA yields of Jurkat and K562 cells were similar between RT experiments with the frozen and fresh cocktails (Fig. 2B). The stability of stored cocktails at $-80^{\circ} \mathrm{C}$ for 1 month allowed us to prepare cocktail plates in bulk, which significantly reduced the reagent cost.

\section{Cost and accuracy of using cocktail plates}

Since the FAS method leverages advantages of robotic capabilities, we note consequent differences between the FAS and SAP protocols (Fig. 1): first, the FAS method used the cocktail plates that were prepared in bulk and stored at $-80^{\circ} \mathrm{C}$; the SAP method used the cocktail plates that were prepared fresh each time prior to the initiation of RT and IVT reactions. Second, the procedure for reagent transferring was different: RT and IVT cocktail plates were used as both reagent source plates and reaction plates in the FAS method, but they were only used as reagent source plates in the SAP method. Of particular note is that the fresh RT cocktail plate for the SAP method contained $14.5 \mu$ l of cocktail, which was $5 \mu$ l more than the volume required for the RT reaction. Since the RT cocktail contains $\sim 10 \%$ glycerol, it could be difficult to accurately transfer $9.5 \mu \mathrm{l}$ of cocktail to the reaction plate from the cocktail source plate using an automation workstation for which the dead volume is less than $5 \mu \mathrm{l}$. With an automation workstation such as the Biomek FX, transferring accuracy increases with increasing dead volume in the cocktail source plate. We selected the dead volume at $5 \mu \mathrm{l}$ as a compromise for the SAP protocol to balance the transfer accuracy and reagent cost. However, it was not practical and cost-effective to have a large dead volume for the preparation of reaction cocktail, particularly for expensive enzyme reagents. Furthermore, additional dead volume was needed each time to prepare the RT cocktail source plate from the cocktail stock solution. The same was true for the IVT cocktail plate used in the SAP method, although the percentage of dead volume was smaller.

In contrast, both RT and IVT cocktail plates for the FAS method were prepared in bulk, so the dead volume was not a factor for the accurate transfer of glycerol-containing cocktails. In fact, the dead volume waste was negligible when multiple cocktail plates were prepared each time. Furthermore, the cocktail stock solution could be prepared more accurately to reflect the intended ratio of each individual component when it was prepared in a large volume. With the FAS transferring procedure, no dead volume was incurred for either the RT or IVT cocktail plates. As indicated in Fig. 1, the transfer of $10 \mu \mathrm{l}$ of RNA sample to the RT cocktail plate and the transfer of $20 \mu \mathrm{l}$ of RT reaction mixtures to the IVT cocktail plate used a "transfer all" procedure. To ensure complete transfer of reagents, we programmed the automation workstation to aspirate materials in the source plate with two times the intended transfer volume by adjusting tip heights, allowing air into the tip to force out all reagents filled, and then to dispense filled reagents with a volume larger than the filled volume to the destination plate. For example, to transfer $10 \mu \mathrm{l}$ of sample from the source plate to the destination plate, the filling volume would be 20 $\mu 1$, and the dispensing volume was set to $30 \mu \mathrm{l}$. This transfer procedure had been proved effective in the FAS method to allow complete transfer of solution from one plate to another plate. On the other hand, the transfer procedure used in the SAP protocol, which depended on the dead volume to ensure accuracy, would not allow any air into the tip during the filling. When air got into the tip (e.g., because of a short dead volume draw or variations of tip positions), the transfer accuracy was compromised. Therefore, the preparation and storage of cocktail plates could reduce the reagent cost by up to $70 \%$ for the RT reaction and $25 \%$ for the IVT reaction; in addition, the reagent transferring procedure used in the FAS ensured accurate volumes of reaction cocktails used in corresponding reactions.

Table 2. Comparison of cRNA Yields of Multiple Sample Plates PROCESSED WITH THE FAS AND SAP

\begin{tabular}{lccccc}
\hline & \multicolumn{2}{c}{ Jurkat cRNA $(\mu g)$} & & \multicolumn{2}{c}{ K562 cRNA $(\mu g)$} \\
\cline { 2 - 3 } \cline { 5 - 6 } Experiment & Mean $\pm S D$ & CV $(\%)$ & & Mean $\pm S D$ & CV $(\%)$ \\
\hline SAP 1 & $50.97 \pm 3.10$ & 7.32 & & $52.49 \pm 4.69$ & 8.33 \\
SAP 2 & $49.31 \pm 2.63$ & 6.33 & & $50.57 \pm 2.05$ & 5.17 \\
FAS 1 & $52.80 \pm 3.98$ & 8.24 & & $51.88 \pm 3.28$ & 7.34 \\
FAS 2 & $47.06 \pm 4.95$ & 8.84 & & $47.63 \pm 4.11$ & 9.64 \\
FAS 3 & $48.10 \pm 4.25$ & 7.61 & & $50.05 \pm 2.08$ & 7.00 \\
FAS 4 & $47.00 \pm 4.14$ & 7.93 & & $47.97 \pm 3.13$ & 7.64 \\
\hline
\end{tabular}

Jurkat and K562 total RNA samples were processed with the FAS and SAP using both fresh cocktails (SAP 1 and FAS 1 ) and cocktails stored at $-80^{\circ} \mathrm{C}$ for 2 weeks (all other experiments). The cRNA yields were presented as mean and $\mathrm{SD}(n=16)$ for each experiment. 


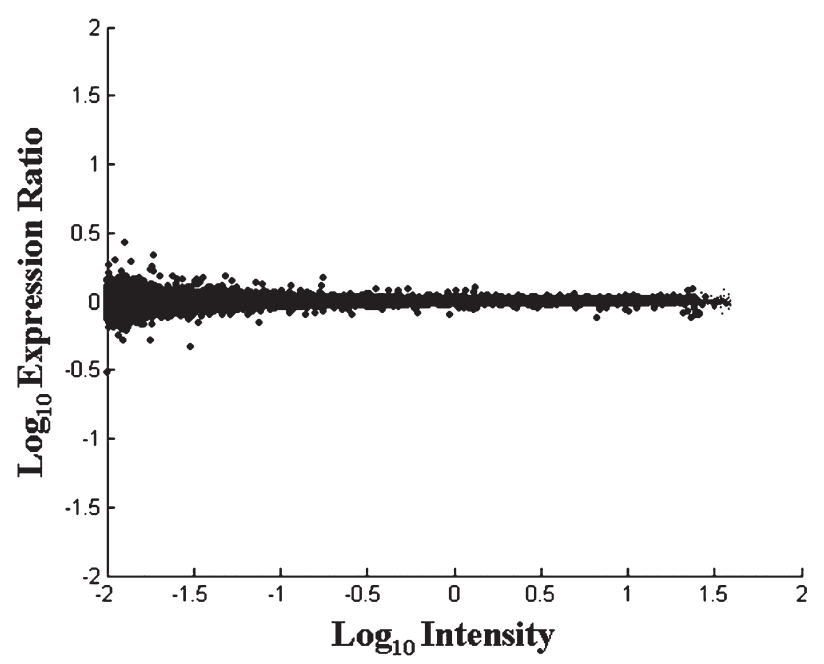

FIG. 3. Reproducibility of cRNA generated on the FAS using stored cocktail plates. The signature plot displayed the results of one pair of the fluor-reversed hybridizations of Jurkat cRNAs from different wells in the same plate from the FAS method. The hybridization signal intensity was plotted versus expression ratio of each gene. A statistical significance was assigned to each gene with black for no change and green or red for down- or up-regulation (if present). Here, there was no significant difference in the expression ratio of genes between these two amplified samples. $P<0.01$.

\section{Processing multiple sample plates using cocktail plates with an FAS}

Preparation of cRNA target from total RNA with the RT-IVT protocol was proved to provide more than sufficient material for microarray hybridization., ${ }^{2,6}$ Actual amplification of mRNA in a total RNA sample could range from 1,000- to 30,000-fold, depending upon the input RNA and number of RT-IVT cycles. ${ }^{2,6-8}$ Because of the amplification factor, robust procedures are needed to mitigate against biases that could lead to artificial expression profiles of genes; in particular, uniform preparation of reagents is one such mechanism to improve robustness. For example, it was our experience that cRNAs amplified within the same experiment by either a manual procedure or SAP were more consistent than those amplified in different experiments even if the input RNA was the same; however, we note that the manual procedure had the largest variation from experiment to experiment or operator to operator, particularly the preparation of reagent cocktails that need to be prepared each time for each experiment. By contrast, multiple plates of RNA samples could be processed with the FAS method in the same experiment with the same set of cocktail plates. As indicated in Table 2, cRNA yields of Jurkat and K562 cells were consistent among four plates from the FAS method and also similar to those from the SAP method. While the fresh and frozen cocktails used for the FAS method were prepared separately in these experiments, the cRNA yields still exhibited low SD. This consistency, we believe in part, resulted from the cocktail preparation in bulk and the elimination of variations in manual operations.

Microarray expression profiling using $c R N A$ amplified with cocktail plates

Under optimized conditions, a high-density oligonucleotide microarray detects differential expression of single-copy genes in many types of cells. ${ }^{1,3}$ While microarray hybridization could reliably measure the differential expression of thousands of genes under the well-controlled process, data consistency may be an issue from one experiment to another. In this study, we focused on two critical questions: (1) was there any differential amplification of the RNA samples from well to well and plate to plate when experiments were performed with reagent cocktails stored at $-80^{\circ} \mathrm{C}$; and (2) could the cRNAs amplified from different RNA samples be used to reliably measure the differential expression in the microarray assay?

Total RNA from two model cell lines of K562 and Jurkat, each in 16 separate wells, were amplified in 96-well plates. One well of Jurkat cRNA was labeled with Cy3 dye or Cy5 dye, and the same was done for another well from either the same plate or different plates, depending on the experimental design. A Cy3-labeled Jurkat cRNA from one well was hybridized with the Cy5-labeled Jurkat cRNA from another well, and vice versa, onto two independent arrays to form fluor-reversed pairs. In this same-versus-same, two-color array experiment, the combined ratio plot of the $\mathrm{Cy} 3$ and $\mathrm{Cy} 5$ channel intensities was expected to be close to 1 (or 0 on the log scale) if there were no difference between the amplified cRNAs. Any significant difference between the labeled cRNAs would be highlighted by the normal ratio plot, indicating inconsistencies in the cRNA amplification from different wells or plates. As we see in Fig. 3, this was not the case; the same-versus-same, two-color array experiment showed that the amplified Jurkat cRNAs from different

Table 3. Detection of Total Regulated Genes in K562 Versus Jurkat Microarray

\begin{tabular}{lcc}
\hline Method & Combined signatures & Mean $\pm S D$ \\
\hline SAP & 8,253 & $8,467 \pm 536$ \\
& 8,071 & \\
& 9,078 & $8,456 \pm 406$ \\
FAS & 8,907 & \\
& 8,344 & \\
& 8,117 &
\end{tabular}

Combined signatures were the sum of up- and down-regulated genes detected in a fluor-reversed microarray experiment with cRNA derived from K562 and Jurkat cells at $P<0.01$ as defined in the error model as described. . $^{3,4}$ 


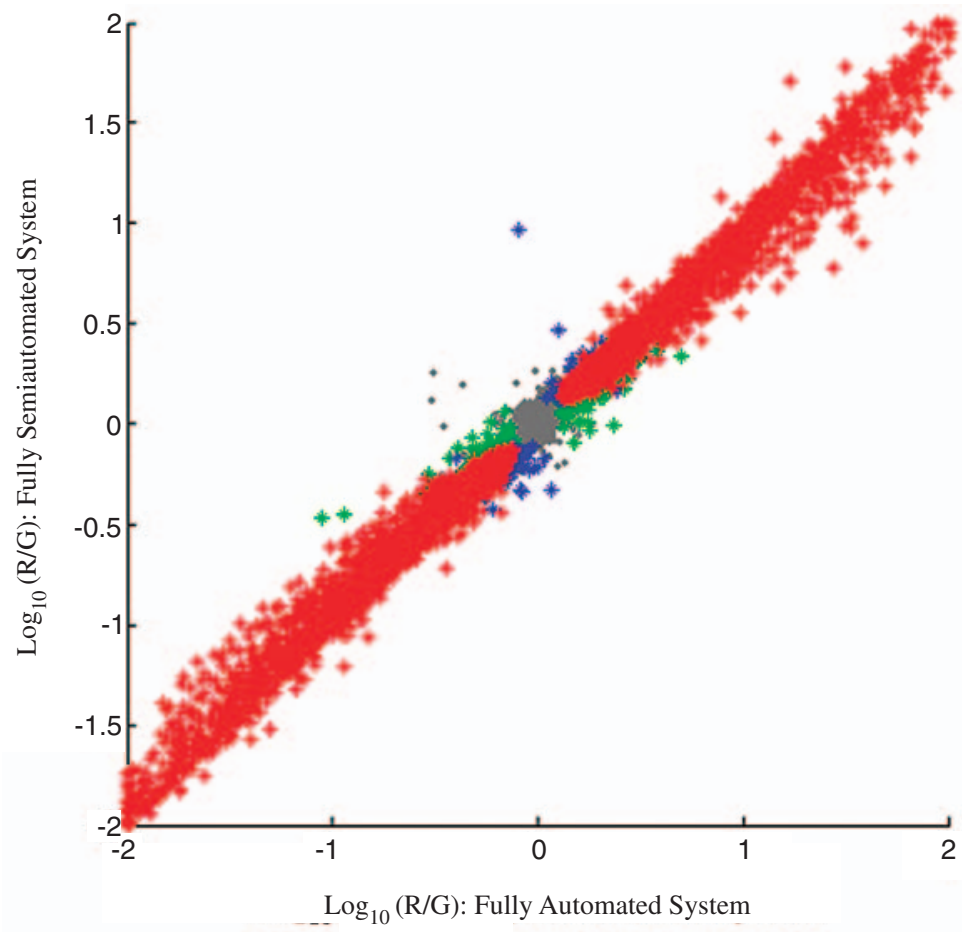

FIG. 4. Correlation of expression ratios for genes in experiments with cRNA generated on the FAS and that on the SAP. The expression ratios for genes in the experiment using Jurkat and K562 cRNA generated on the FAS were plotted against the expression ratios for genes in the experiment using Jurkat and K562 cRNA generated on the SAP. Genes correlated (signatures, either induced or repressed) are in red, genes detected as signatures by the FAS method but not the SAP method are in green, genes detected as signatures by the SAP method but not the FAS method are blue, and genes unchanged are in gray. $P<0.01$; correlation $($ all dots $)=0.99 ;$ correlation $($ signature $)=0.99$; detection limit $=-1.5$; number of good spots $=$ 23,224 ; number of good spots less than the detection limit $(-1.5)=15,099$; number of correlated spots $=7,230$; number of anti-correlated spots $=$ 0 ; number of correlated in $x$ spots $=715$; number of correlated in $y$ spots $=529$. R/G, red/green. wells or different plates on the FAS were virtually the same: no genes were identified as differentially regulated, and there was little variation around $\log$ ratio equal to zero across the entire intensity range of the ratio plot at $P<0.01$. Repeated runs of these experiments produced the same results, illustrating the high degree of reproducibility of the cRNA amplification by the FAS method.

Next, two different total RNA samples (Jurkat and K562) were labeled and hybridized in fluor-reversed pairs to address the question whether the cRNAs amplified with the FAS could reliably detect differential gene expression. Differentially detected genes from the combined signatures would be a measurement of the ability to profile two disparate RNA samples. As shown in Table 3 , the detectable total number of signature genes in the experiment with cRNAs of Jurkat and K562 cells from the FAS method was similar to those from the SAP method. Gene expression ratios detected in Jurkat versus K562 hybridization from the FAS method were highly correlated with those from the SAP method (correlation coefficient of 0.99 at $P<0.01$, Fig. 4). The calculated concordance of signature genes measured by both protocols was $93 \%$. Furthermore, receiver operating characteristic (ROC) plots were used to compare the sensitivity and specificity of the microarray hybridization of cRNAs amplified with two different methods; the number of false-positives on the $x$-axis indicates specificity (data derived from Jurkat versus Jurkat hybridizations), while the number of positives on the $y$-axis indicates sensitivity (data derived from Jurkat versus K562 hybridizations). ${ }^{9}$ The ROC analysis revealed similar sensi- tivity and specificity of these two methods with which cRNAs were amplified with reagents prepared under indicated conditions (Fig. 5). As shown in Fig. 4, the total signatures were $7,945(7,230+715)$ from the FAS and

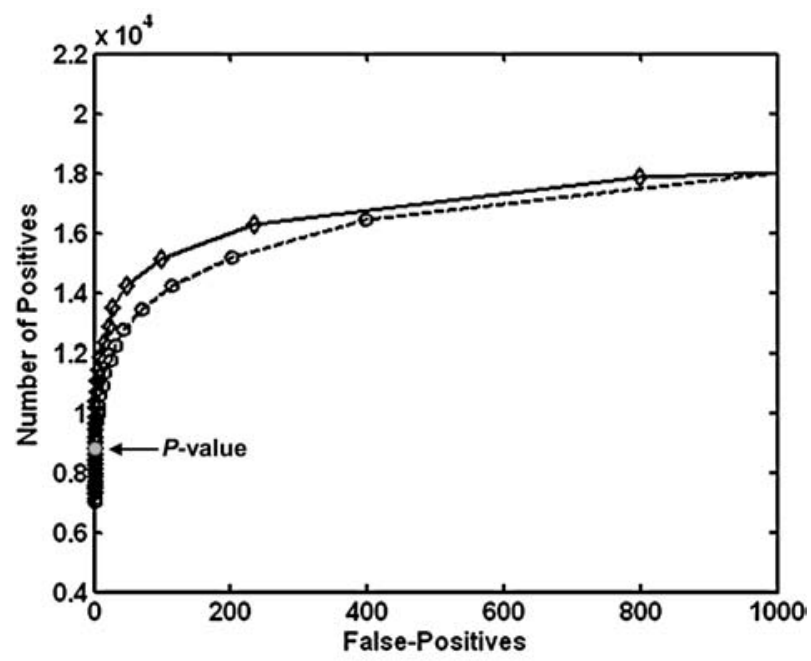

FIG. 5. Sensitivity and specificity of microrrays with cRNA generated on the FAS and the SAP. The ROC curves demonstrated the sensitivity and specificity of microarray hybridizations that used the cRNAs prepared with the FAS $(\diamond)$ and SAP (O). The number of false-positives on the $x$-axis indicated the specificity (data derived from Jurkat versus Jurkat cell arrays); the number of positives on the $y$-axis indicated the sensitivity (data derived from Jurkat versus K562 cell arrays). Each ROC curve was generated with data from one pair of Jurkat versus Jurkat cell arrays against data from one pair of Jurkat versus K562 cell arrays, and one representative ROC curve of three plots was shown for each method. $P=0.01$ ( $\bigcirc$ ). 
$7,759(7,230+529)$ from the semiautomated system for a cutoff of $P<0.01$. The maximal difference in total signatures between these two systems was 186 at $P<0.01$, which was less than $1 \%$ from the total 23,224 gene sequences on the array. This result demonstrated that cRNAs generated from the FAS could reliably reproduce the differential gene expression generated by manual or SAP methods from Jurkat and K562 RNA samples.

\section{Summary}

A method of preparing and storing the ready-to-use RT and IVT cocktail plates has been developed. The stored cocktail plates retained their enzymatic activity at $4^{\circ} \mathrm{C}$ for up to $24 \mathrm{~h}$ following storage at $-80^{\circ} \mathrm{C}$ for at least a period of 1 month. These cocktail plates were fully functional for nucleic acid amplification by RT and IVT as determined by cRNA or cDNA yield in comparison with fresh cocktails. The bulk preparation of reagent plates and the method for storage of reagent plates enabled the FAS to perform the complicated RT-IVT procedure for simultaneously amplifying multiple plates of cDNA and cRNA targets. We showed data that indicated that prealiquoting bulk preparation of reagents could introduce robustness in this complex, multistep protocol by reducing variance introduced by manual preparation of reagents immediately prior to use. Microarray data showed the cRNA generated with the stored cocktails could reliably measure the differential gene expression of different total RNA samples. The application of the reagent storage method and the optimized transfer procedure on the FAS has been proved to be a cost-effective and high-throughput method for the amplification of microarray hybridization targets.

\section{Acknowledgments}

We thank Dr. Matthew J. Marton for his critical review of the manuscript.

\section{References}

1. Hughes TR, Shoemaker DD: DNA microarrays for expression profiling. Curr Opin Chem Biol 2001;5:21-25.

2. Phillips J, Eberwine JH: Antisense RNA amplification: a linear amplification method for analyzing the mRNA population from single living cells. Methods 1996;10:283-288.

3. Hughes TR, Mao M, Jones AR, Burchard J, Marton MJ, Shannon KW, et al.: Expression profiling using microarrays fabricated by an ink-jet oligonucleotide synthesizer. Nat Biotechnol 2001;19:342-347.

4. Marton MJ, DeRisi JL, Bennett HA, Iyer VR, Meyer MR, Roberts CJ, et al.: Drug target validation and identification of secondary drug target effects using DNA microarrays. Nat Med 1998;4:1293-1301.

5. Ma M, Lyons-Weiler M, Liang M, LaFramboise W, Gilbertson JR, Becich MJ, et al.: In vitro transcription amplification and labeling methods contribute to the variability of gene expression profiling with DNA microarrays. $J \mathrm{Mol} D i$ agn 2006;8:183-192.

6. Rajeevan MS, Dimulescu IM, Vernon SD, Verma M, Unger ER: Global amplification of sense RNA: a novel method to replicate and archive mRNA for gene expression analysis. Genomics 2003;82:491-497.

7. Yim SH, Ward JM, Dragan Y, Yamada A, Scacheri PC, Kimura S, et al.: Microarray analysis using amplified mRNA from laser capture microdissection of microscopic hepatocellular precancerous lesions and frozen hepatocellular carcinomas reveals unique and consistent gene expression profiles. Toxicol Pathol 2003;31:295-303.

8. Polacek DC, Passerini AG, Shi C, Francesco NM, Manduchi E, Grant GR, et al.: Fidelity and enhanced sensitivity of differential transcription profiles following linear amplification of nanogram amounts of endothelial mRNA. Physiol Genomics 2003;13:147-156.

9. Khodarev NN, Park J, Kataoka Y, Nodzenski E, Hellman S, Roizman B, et al.: Receiver operating characteristic analysis: a general tool for DNA array data filtration and performance estimation. Genomics 2003;81:202-209.

Address reprint requests to: Mingjie Zhou, Ph.D.

Rosetta Inpharmatics LLC 401 Terry Avenue $N$ Seattle, WA 98109

E-mail: Mingjie_Zhou@Merck.com 